\title{
Análisis de la Prevención Podológica. Un estudio a través de Internet
}

\author{
Analysis of Podiatric Prevention. A study throught Internet
}

\author{
Daniel López López ${ }^{1}$, Ricardo García Mira ${ }^{2}$, Francisco Alonso Tajes ${ }^{1}$, \\ Luis LóPEz LóPEz ${ }^{1}$
}

\begin{abstract}
${ }^{1}$ Licenciado en Podología. Profesor Podología E.U Enfermaría e Podoloxía Departamento de Ciencias da Saúde. Universidade da Coruña

${ }^{2}$ Doctor en Piscología. Profesor Titular Facultad de Ciencias de la Educación Departamento de Psicología. Universidade da Coruña
\end{abstract}

Correspondencia:

Prof. Daniel López López

Universidade da Coruña

Departamento de Ciencias da Saúde.

E.U Enfermaría e Podoloxía.

c/ Naturalista López Seoane. Esquina San Ramón s/n

Campus Universitario de Esteiro

15403 Ferrol

E-mail: daniellopez@udc.es

Fecha de recepción: 24 enero de 2012

Fecha de aceptación: 5 marzo de 2012

Los autores declaran no tener ningún tipo de interés económico o comercial.

\section{RESUMEN}

La existencia en la sociedad actual de un gran número de enfermedades, inicialmente no relacionadas con patologías en los pies (por ej. diabetes, obesidad, trastornos cardiovasculares, estrés..), con potencial para mermar la calidad de vida del paciente, ha favorecido la aparición de conductas eventualmente generadoras de riesgo de enfermedad en los pies.

En la medida en que tales enfermedades suponen transformaciones importantes en la morfología estructural del cuerpo en general, y del pie en particular, plantean demandas que, a su vez, requieren la adopción de medidas concretas de intervención podológica.

En este artículo se describen los resultados de un estudio cuantitativo llevado a cabo a través de internet por medio de una aplicación telemática que evalúa conductas generadoras de riesgo de la salud del pie.

Los resultados hacen hincapié en la importancia de los estudios relacionados con la percepción social del podólogo y de las patologías de los pies, así como en la necesidad de incorporar información subjetiva al análisis de la problemática del pie y de aquellos comportamientos que implican riesgos para la salud podal del ser humano. Esta perspectiva contribuye a la realización de análisis en profundidad del significado social de la podología, y el modo mejor de afrontar el cada vez mayor número de enfermedades que pueden mermar la calidad de vida, debido a la persistencia de determinadas conductas, potencialmente generadoras de riesgo de enfermedades en los pies.

Palabras clave: Salud; Cuidado del Pie; Patologías en los pies; Bienestar; Calidad de vida.

\section{ABSTRACT}

The existence in today's society a large number of diseases, initially unrelated to foot pathology (eg. Diabetes, obesity, cardiovascular disorders, stress...), with the potential to diminish the quality of life for patients, has resulted the emergence of behavior eventually generating disease risk in the feet. 
To the extent that these diseases represent major changes in the structural morphology of the body in general and in particular standing pose demands, in turn, require the adoption of concrete measures podiatric intervention.

In this article, results of a quantitative study carried out with 200 patients of both genders, without restrictions of age who attend a podiatry clinic, are described.

For collecting data, a questionnaire ad hoc was designed. It allowed to obtain accurate information about the profile of the patient, in general, and of the specific characteristics of that profile: socio-demographical, behavior linked to lifestyles, everyday habits, subjective relevance, preventing behaviours, as well as perception and motivation.

Results focus in the relevance of social perception studies of podologist and foot diseases, as well as in the need of incorporating subjective information in the analysis of the foot problems, and those behaviours involving risks for foot health of the human beings. This perspective contribute to the deeper analysis of the social meaning of podology, and the best way to deal with the increasing number of diseases that can reduce the quality of life, due the persistence of some behaviours, potentially generating risk of foot diseases.

Key words: Health; Foot Care; Foot disorders; Wellbeing; Life quality.

Sumario: 1. Introducción, 2. Objetivos generales de la investigación, 3. Método, 4. Resultados, 5. Discusión, 6. Conclusiones, Bibliografía.

Referencia normalizada: López López, D., García Mira, R., Alonso Tajes, F., López López, L. Análisis de la Prevención Podológica. Un estudio a través de Internet. Rev. Int. Cienc. Podol. 2012; 6(2): 63-72. 


\section{INTRODUCCIÓN}

Se ha señalado que el impacto del contexto psicosocial en la respuesta al tratamiento que rodea a una intervención contribuye a resultados positivos terapéuticos ${ }^{1}$. La necesidad de estudiar este contexto, caracterizado por el desarrollo de actividades de diversa índole, que las personas llevan a cabo en su vida habitual, constituye un objetivo de esta investigación. Al mismo tiempo, se sabe que la importancia atribuida a la enfermedad y la atribución, en general, que las personas hacen de sus causas posee una influencia sobre el tipo de conductas de prevención que acompañan a un tratamiento o que reducen la posibilidad de sufrir una patología podal.

$\mathrm{Si}$ atendemos a la prevención que desarrolla el profesional, nos encontramos con que la prevención primaria que ejerce el podólogo está enfocada a evitar la aparición de la enfermedad en general, y del pie en particular, por medio de actividades educacionales y de diagnóstico precoz selectivo en pacientes con enfermedades de base como la Artritis Reumatoidea, Cardiopatías, Diabetes, Estrés u Obesidad. Incluso en el caso de complicaciones del pie causadas por diabetes, hay una asociación con el tipo de tratamiento preventivo que se haga respecto del control metabólico ${ }^{2}$ y su efecto de reducción de posibles complicaciones microvasculares, de reducción de factores de riesgo cardiovasculares que reducen la susceptibilidad a la isquemia provocada por una enfermedad macrovascular ${ }^{3}$.

La prevención secundaria que ejerce el podólogo está enfocada a detectar la enfermedad al principio de su evolución. Los programas de promoción de de la salud dirigidos específicamente a un grupo poblacional proporcionan información apropiada para que las personas comprendan mejor la problemática de las patologías del pie en particular y del estado físico y psicológico en general, recalcando la importancia de estos programas en:

a) Etapa Infantil. La calidad de vida en los niños es un elemento clave diferenciador de las sociedades que disfrutan de políticas basadas en el bienestar general de la población, y del pie en particular. Los pies, por otro lado, además de fa- cultarnos para el ejercicio de caminar, proporcionan una base importante en la organización mental de la información social y espacial. Para García-Mira, Goluboff, García-Fontán y Romay ${ }^{4}$ y García-Mira y Goluboff 5 , este recurso que utilizamos para desplazarnos facilita la adquisición del conocimiento ambiental y la oportunidad de vivenciar el ambiente e integrarlo cognitivamente. Cuando esto no se prevé o se ve alterado por un trastorno podal y se recorren distancias en el coche el efecto sobre la organización cognitiva de la información espacial es más pobre influyendo de manera significativa en la percepción de calidad de vida.

b) Tercera Edad: al implementar programas preventivos en este grupo poblacional sobre los trastornos del pie y la pierna y de sus potenciales efectos sobre la movilidad y la independencia, permiten una mejor gestión de sus problemas de salud en los pies y en las piernas y contribuyen a mejorar la limitación funcional y reducir los riesgos de la aparición de una discapacidad en el pie o en el miembro inferior ${ }^{6,7}$.

c) Diabéticos: para Reiber ${ }^{8}$ el riesgo de ulceración en el pie en los pacientes diabéticos oscila entre el $1 \%$ y $4 \%$, por esta razón, es prioritario educar a las personas con diabetes en el control de la enfermedad, la prevención de complicaciones, proteger sus pies de un traumatismo menor, el reconocimiento de signos de ulceración incipiente y la reducción del riesgo de desarrollar úlceras. De esta manera, el riesgo de ulceración y amputación puede reducirse ${ }^{9}$.

En cuanto a la prevención terciaria que ejerce el podólogo, su enfoque va a ir encaminado a limitar las complicaciones y la discapacidad debidas a la enfermedad presente.

En este contexto, las creencias y atribuciones causales que los pacientes hacen son un elemento clave. Para López, García-Mira, Alonso y López ${ }^{10}$, las creencias que presentan las personas en relación a los factores que contribuyen a la aparición de enfermedades en los 
pies, tienden a estar relacionadas con su labor profesional, pero también con los traumatismos, el sobrepeso, y también con el calzado que utiliza. Estudiar las creencias nos permite saber si los conocimientos y percepciones que tiene el enfermo coinciden o no con las características de la enfermedad. Desde este punto de vista, las creencias adquieren un papel clave en la participación del paciente en el autocuidado de sus pies. Veamos los grupos poblacionales con mayor exposición a la enfermedad:

a) Población General: el rol del podólogo en el cuidado de la salud en general y del pie en particular permite a los pacientes que acuden al podólogo, adquirir confianza, así como una mayor implicación en la gestión del riesgo de la salud del pie y una búsqueda de un cambio de conductas individuales para la promoción de la salud ${ }^{3,10}$.

Otro aspecto importante, que no será abordado aquí, pero sí debe reflejarse por el papel que desempeña, es la evaluación de la autoeficacia percibida ${ }^{11}$, cuya exploración permite acceder al conocimiento sobre el control que el paciente tiene acerca de su enfermedad.

Para estos autores, los pacientes que no seguían el consejo médico o el plan terapéutico establecido tendían a tener puntos negativos de la enfermedad y de las limitaciones que imponían a su estilo de vida, haciendo lo que consideraran oportuno al creer que estaban sanos, ocultando su verdadera conducta para evitar respuestas negativas por parte de sus mé$\operatorname{dicos}^{12,13}$. Por el contrario, los pacientes que seguían la pauta establecida eran los más satisfechos con su enfermedad y con la comunicación con los profesionales sanitarios ${ }^{14}$.

b) Población Infantil: el análisis de las percepciones de salud en la etapa infantil, en niños con patologías en los pies permite conocer los síntomas físicos y las limitaciones que generan restricciones en la participación y en su desarrollo social.

Para Morris ${ }^{15}$ la educación va a permitir modificar las actitudes y las conductas y de esa manera mejorar la experiencias del niño relacionadas con la autoestima y la percepción del ambiente.

La flexibilidad de las reglas de la escuela y la inclusión de los niños en las actividades grupales les permite evitar la frustración y obtener una mayor consideración y cooperación social.

Estas experiencias positivas facilitan niños más creativos y con menos dificultades en el control de su autonomía y el desempeño funcional en todos los ambientes de la vida diaria ${ }^{16}$.

c) Pies de Riesgo: el papel del podólogo en el tratamiento de las úlceras en el pie en pacientes con diabetes o artritis contribuye a evitar complicaciones, siendo la prevención terciaria aquí un elemento de control de la salud en general y del pie en particular ${ }^{8}$.

d) Senectud: en esta etapa, el estado anímico y las experiencias positivas de los ancianos contribuyen de manera significativa al mantenimiento de la salud y permiten evitar la involución sensorial, motriz e intelectual más acentuada en este grupo poblacional. Si no fuera de ese modo, se generaría un empobrecimiento de la vida afectiva, de los contactos sociales o de la propia autonomía, imprimiendo una mayor dificultad a la adaptación a situaciones nuevas, potenciando un sentimiento de inseguridad y repercutiendo de manera negativa en la calidad de vida ${ }^{17}$.

En resumen, la prevención de las diferentes enfermedades conocidas, que tienen una mayor incidencia y repercusión en el pie, posibilita fomentar conductas saludables relacionadas con los hábitos comportamentales que conferirán en los pacientes una vida sana, autónoma y alejada de situaciones de dependencia.

Desde este punto de vista, la realización del presente estudio intenta proporcionar información sobre la percepción social de las patologías podales, de modo que pueda servir de base para la elaboración de perfiles de conductas preventivas, actividades, importancia atribuida a la profesión podológica y atribución de causas por parte de la población en general. 


\section{OBJETIVOS GENERALES DE LA INVESTIGACIÓN}

El objetivo general consistió en realizar un laboratorio web ${ }^{18,19}$ para asi evaluar los determinantes de salud que puedan tener repercusión en la aparición de patologías en los pies y así prevenir conductas generadoras de riesgo para la salud del pie.

Los objetivos específicos son: 1) Conocer la prevención que realiza la población general para evitar patologías en el pie; 2) Valorar las percepciones relacionadas con las patologías en los pies.

La necesidad de evaluar el pie y predecir ciertas conductas inadecuadas evitará la aparición de ciertos trastornos que están asociados al dolor, discapacidad y la merma de la calidad de vida y contribuir a un cuidado óptimo de los pies y de la salud en general.

\section{MÉTODO}

\section{Muestra}

La muestra estuvo compuesta por 300 personas usuarios de internet, de los cuales el $65 \%$ son mujeres y el $35 \%$ hombres.

\section{Procedimiento}

Para la recogida de datos se diseñó cuestionario ad hoc que permitió obtener información precisa acerca de las características sociodemográficas del usuario que utiliza internet y de las conductas subjetivas vinculadas a la prevención de la salud del pie.

Para ello se desarrollo una aplicación informática que permitió alojar el cuestionario (véase Fig.1) en el en el espacio web http://investigacionpodo.blogspot.com/ (véase Fig.2)

El bloque de datos que analizaremos aquí abarca un conjunto de 25 ítems, que midieron las variables antes mencionadas, en varios tipos de escalas: 1) Escalas cualitativas, con ítems abiertos que recogieron información sobre conductas preventivas relacionadas con la salud del pie; 2) Escalas tipo Likert de 5 puntos, para medir la importancia atribuida por los sujetos a la salud de los pies en general, y al profesional de la podología y su situación en el sistema de salud, en particular.

\section{Análisis de datos}

Teniendo en cuenta las clasificaciones y las características de los distintos métodos de investigación, el método descriptivo, basado en el paradigma cuantitativo, será en el que se centre nuestra investigación.

La propia aplicación informática nos permitió obtener tablas y diagramas de frecuencias, ya que nuestro principal objetivo es describir sistemáticamente hechos y características de los usuarios de internet relacionados con la salud del pie.

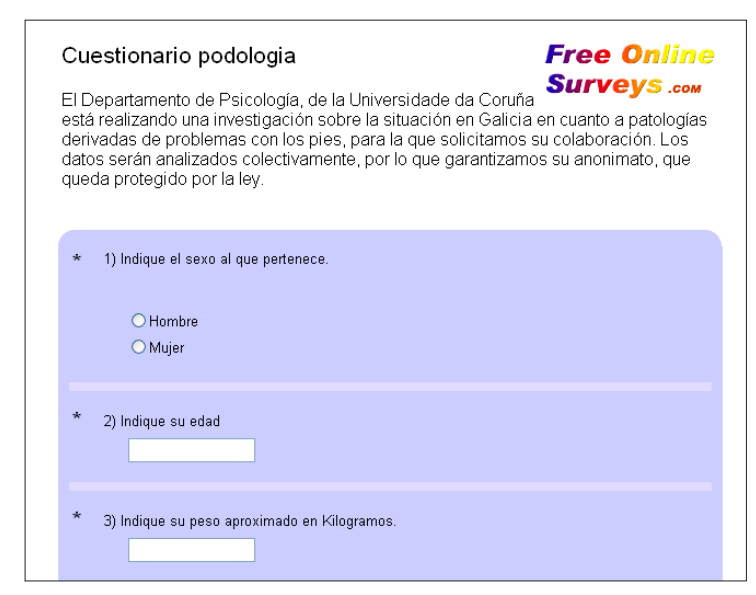

Figura 1. Cuestionario.

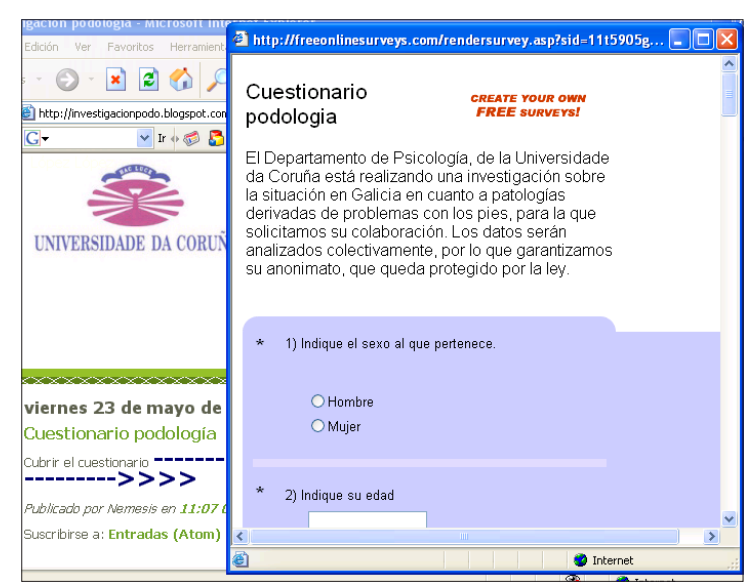

Figura 2. Espacio Web. 


\section{RESULTADOS}

Los resultados obtenidos en el cuestionario online muestran que el $55 \%$ de las personas que lo contestan son universitarias (véase Fig. 3) y que la edad de los participantes corresponde a la etapa adulta temprana, con una media de edad de 26 años, esto es debido al mayor número de usuarios que utilizan internet en este rango de edad, para el Estudio General de Medios $(E G M)^{20}$ el 59,4\% de la población española manejan internet de manera habitual.

Por ende, Internet se ha convertido en una de las actividades complementarias preferida por la gran mayoría de los encuestados (véase Tabla 1).

El sexo predominante es el femenino con $65.1 \%$ frente al $34.9 \%$ de hombres que contestan dicho experimento podológico. La mujer parece mostrar en este estudio una tendencia mayor a responder este tipo de cuestionarios. En general, la mujer en todo lo relacionado con la salud está más comprometida, y se muestra más interesada. Si atendemos a Chesterton, Barlas, Foster, Baxter y Wright ${ }^{21}$ y a Wise, Price, Myers, Heft, y Robinson ${ }^{22}$, quizá esto esté en relación con la menor tolerancia de la mujer al dolor de los pies en particular, razón por la cual consultan antes y experimentan dolor durante menos tiempo que los hombres.

El $45 \%$ de las personas admiten consumir tabaco habitualmente con una media de 0.9 cajetillas semanales, el $55 \%$ de la muestra estudiada ingiere una media de 5 copas semanales y el $65 \%$ consumen 1.5 cafés diarios.

En cuanto a las percepciones relacionadas con las enfermedades del pie, los encuestados tienden a considerar como factor principal de

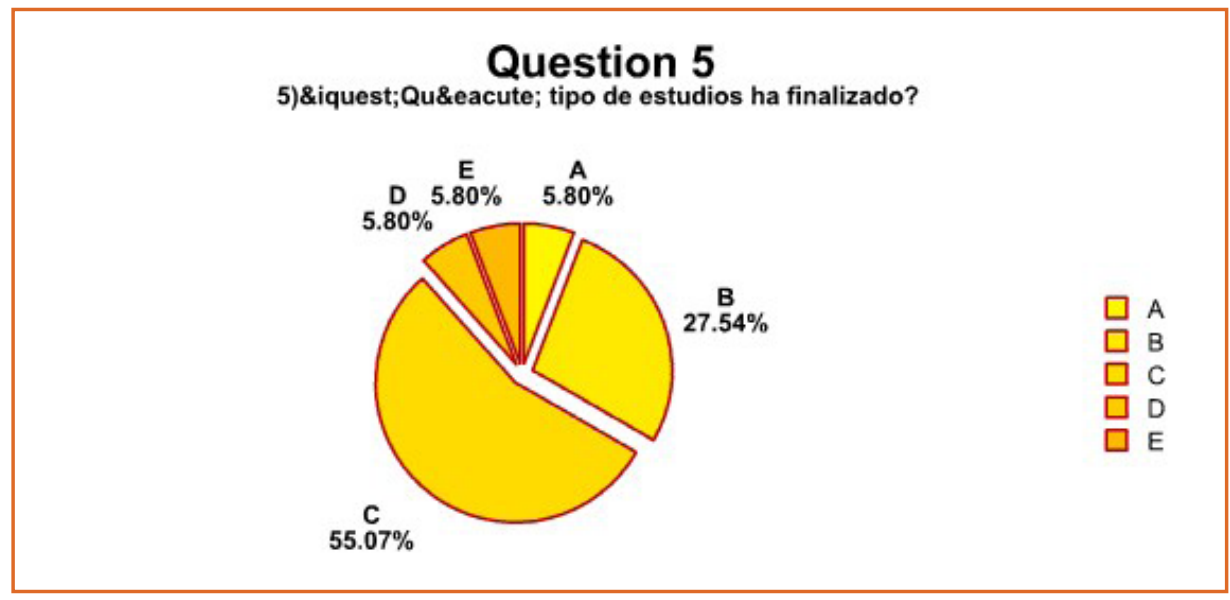

Figura 3. Nivel de Estudios.

\begin{tabular}{lccccc} 
& Nada & Algo & Poco & Bastante & Mucho \\
\hline Bailar & $(29.51 \%)$ & $\mathbf{( 3 2 . 7 9 \% )}$ & $(11.48 \%)$ & $(24.59 \%)$ & $(1.64 \%)$ \\
Ejercicio físico & $(17.46 \%)$ & $(17.46 \%)$ & $\mathbf{( 2 6 . 9 8 \% )}$ & $(22.22 \%)$ & $(15.87 \%)$ \\
\hline Escuchar música & $(3.17 \%)$ & $(15.87 \%)$ & $(11.11 \%)$ & $\mathbf{( 3 8 . 1 0 \% )}$ & $(31.75 \%)$ \\
\hline Leer & $(9.52 \%)$ & $(14.29 \%)$ & $(20.63 \%)$ & $\mathbf{( 4 4 . 4 4 \% )}$ & $(11.11 \%)$ \\
\hline Pasear & $(6.45 \%)$ & $(17.74 \%)$ & $(32.26 \%)$ & $\mathbf{( 3 8 . 7 1 \% )}$ & $(4.84 \%)$ \\
\hline Utilizar Internet & $(4.92 \%)$ & $(4.92 \%)$ & $(13.11 \%)$ & $\mathbf{( 4 0 . 9 8 \% )}$ & $(36.07 \%)$ \\
\hline Ver la tv, dvd, videos & $(8.06 \%)$ & $(19.35 \%)$ & $\mathbf{( 3 7 . 1 0 \% )}$ & $(19.35 \%)$ & $(16.13 \%)$ \\
\hline
\end{tabular}

Tabla 1. Actividades Complementarias. 
causalgia el calzado (véase Tabla 2), existiendo numerosos estudios que también relacionan el uso de un calzado no adecuado con la aparición de lesiones en los pies, aparición de inestabilidad en la marcha, aumento del riesgo de sufrir caídas y descenso de la independencia del sujeto $^{23,24,25}$.

Del mismo modo, la conducta más repetida por los encuestados para prevenir las patologías podológicas se enfoca hacia la higiene con un
$71,4 \%$ (véase Tabla 3), Teixeira ${ }^{26}$ recalca la importancia del cuidado y de las conductas preventivas para que la vida no se vea alterada. De ahí la preocupación de las posibles consecuencias que mermarían de manera significativa su calidad de vida.

Los usuarios perciben la necesidad del cuidado del pie. El 57\% lo consideran muy importante (véase Fig. 4). Con bastante probabilidad, ésto se debe al aumento de enfermedades cróni-

\begin{tabular}{lccccc}
\hline & Nada & Algo & Poco & Bastante & Mucho \\
\hline Deporte & $(17.46 \%)$ & $(19.05 \%)$ & $(12.70 \%)$ & $\mathbf{( 3 8 . 1 0 \% )}$ & $(12.70 \%)$ \\
\hline Enfermedad de Base & $(19.05 \%)$ & $(12.70 \%)$ & $(9.52 \%)$ & $\mathbf{( 4 7 . 6 2 \% )}$ & $(11.11 \%)$ \\
\hline Infecciones & $(9.52 \%)$ & $(9.52 \%)$ & $(23.81 \%)$ & $\mathbf{( 4 7 . 6 2 \% )}$ & $(9.52 \%)$ \\
\hline Sobrepeso & $(9.52 \%)$ & $(12.70 \%)$ & $(4.76 \%)$ & $\mathbf{( 4 9 . 2 1 \% )}$ & $(23.81 \%)$ \\
\hline Traumatismo & $(6.35 \%)$ & $(4.76 \%)$ & $(9.52 \%)$ & $\mathbf{( 5 2 . 3 8 \% )}$ & $(26.98 \%)$ \\
\hline Trabajo & $(7.94 \%)$ & $(3.17 \%)$ & $(17.46 \%)$ & $\mathbf{( 5 7 . 1 4 \% )}$ & $(14.29 \%)$ \\
\hline Calzado & $(4.76 \%)$ & $(1.59 \%)$ & $(6.35 \%)$ & $(39.68 \%)$ & $\mathbf{( 4 7 . 6 2 \% )}$ \\
\hline
\end{tabular}

Tabla 2. Factores Desencadenante de Enfermedades.

\begin{tabular}{lccccc} 
& Nunca & Alguna vez & Pocas veces & Bastantes veces & Muchas veces \\
\hline Acudir al podólogo & $\mathbf{( 4 2 . 8 6 \% )}$ & $(28.57 \%)$ & $(9.52 \%)$ & $(6.35 \%)$ & $(12.70 \%)$ \\
Autotratamientos & $\mathbf{( 5 2 . 3 8 \% )}$ & $(22.22 \%)$ & $(11.11 \%)$ & $(11.11 \%)$ & $(3.17 \%)$ \\
\hline Calzado & $(6.35 \%)$ & $(11.11 \%)$ & $(19.05 \%)$ & $\mathbf{( 3 8 . 1 0 \% )}$ & $(25.40 \%)$ \\
\hline Higiene & $(1.59 \%)$ & $(1.59 \%)$ & $(4.76 \%)$ & $(20.63 \%)$ & $\mathbf{( 7 1 . 4 3 \% )}$ \\
\hline Hidratación & $(11.11 \%)$ & $(15.87 \%)$ & $\mathbf{( 2 8 . 5 7 \% )}$ & $(19.05 \%)$ & $(25.40 \%)$ \\
Secado & $(4.76 \%)$ & $(7.94 \%)$ & $\mathbf{( 3 1 . 7 5 \% )}$ & $(28.57 \%)$ & $(26.98 \%)$ \\
\hline
\end{tabular}

Tabla 3. Pautas Preventivas del Cuidado del Pie.

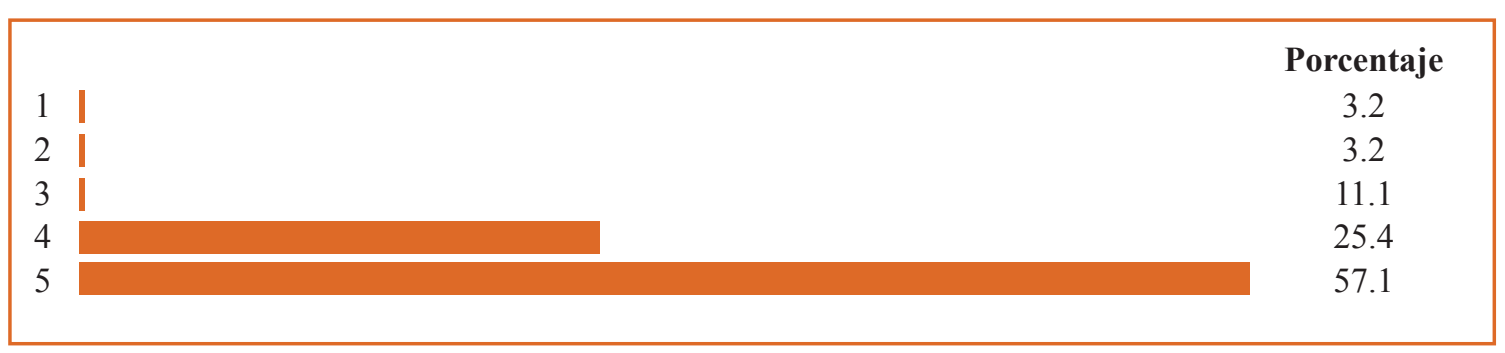

Figura 4. Importancia del Cuidado del Pie. 
cas que se caracterizan por su origen multifactorial, donde el comportamiento y el estilo de vida individual juegan un papel fundamental en su desarrollo, y donde la prevención podológica aparece como una opción para mejorar la calidad de vida y la autonomía del paciente ${ }^{17}$.

\section{DISCUSIÓN}

En la última década, el auge de internet ha permitido que algunos investigadores hayan optado por colocar sus experimentos en páginas web y esperar a que aparezcan usuarios dispuestos a participar en ellos. Varias universidades han optado ya por este medio para captar y difundir información susceptible de ser analizada, y cuentan con una sección especial para este fin ${ }^{18}$.

El presente trabajo constituye una aproximación a las conductas generadoras de riesgo para la salud del pie desde una perspectiva de intervención podológica, con orientación psicosocial, analizando las conductas de protección y prevención de la salud del pie.

La intervención podológica, a nivel general, evalúa los determinantes de salud. En este estudio se ha puesto en relación con la aparición de hábitos de vida poco saludables que están condicionados por el estilo de vida que corresponde al sendentarismo asociado al uso de internet, consumo de tabaco, alcohol y café, que configura un patrón comportamental a destacar como punto de inicio precoz, aunque subclínico, de enfermedades crónicas que pueden tener repercusión en el pie. Para Mokdad et. al., ${ }^{27}$ este patrón comportamental es el responsable de alrededor de 400.000 muertes cada año en los Estados Unidos.

La representación mayoritaria en este estudio del género femenino, también está relacionada con el mayor porcentaje de personas que utilizan internet, según la EMC de $2010^{28}$.

Las características sociodemográficas aparecen ligadas a la etapa universitaria, con una media de edad de 26 años. Para Ruíz ${ }^{29}$ este patrón de comportamiento y su repercusión fisiológica se fija principalmente durante la etapa de la adolescencia, debido a los cambios psicológicos que tienden a afectar a la imagen corporal, la forma de alimentarse y el modo de comportarse, que suelen persistir durante muchos años o incluso durante toda la vida.

En cuanto a los objetivos específicos relacionados con la prevención y la percepción de la salud del pie, podemos afirmar que las personas que han cumplimentado el experimento podológico, llevan a cabo conductas normativas de prevención de la salud del pie, basadas en la higiene diaria y en la utilización de un calzado óptimo.

La educación terapéutica se mantiene como el eslabón más débil en la cadena de la prevención del cuidado del pie. Obviamente, ésto es debido a la carencia de información clínica de las personas que no acuden a una revisión podológica periódica. De este modo, se impide el desarrollo de habilidades para la toma de decisiones diarias que influyan en conductas generadoras de autocuidado diario y beneficioso para los pies.

Las creencias por parte de las personas que han participado en este estudio, en relación a los factores que contribuyen a la aparición de enfermedades en los pies, tienden a estar relacionadas con el calzado y con la situación laboral. Esto nos ha permitido saber si conocimientos y percepciones que tiene el usuario coinciden o no con las características de la enfermedad, desempeñando un papel clave en la participación del paciente en el autocuidado de sus pies.

\section{CONCLUSIONES}

La necesidad de realizar estudios de evaluación psicológica y de analizar aquellos comportamientos que introducen riesgos para la salud podal del ser humano, reclama un mayor número de contribuciones en el ámbito de la podología. En la actualidad, la alta prevalencia de enfermedades crónicas, en la mayoría de los países, para los cuales no existe una curación total y donde el objetivo del tratamiento es atenuar o eliminar síntomas, evitar complicaciones y mejorar el bienestar de los pacientes, lleva a que las medidas clásicas de resultados en medicina (mortalidad, morbilidad, expectativa de vida) no sean suficientes para evaluar de manera integral, si los pacientes reciben un tratamiento apropiado y eficaz a nivel de las enfermedades del pie. 
Si a lo anteriormente expuesto, se le añade la existencia de un gran número de enfermedades, inicialmente no relacionadas con patologías en los pies (por ej. diabetes, obesidad, trastornos cardiovasculares, estrés..), con potencial para mermar la calidad de vida del paciente, podemos entender la premura en el estudio de aquellas conductas eventualmente generadoras de riesgo de enfermedad en los pies.

El abordaje de una investigación desde una vertiente más actual, basada en el estudio de conductas de riesgo, supone una clarificación de aquellos aspectos que permiten la utilización de cuestionarios como herramienta básica para la obtención de información y la definición de perfiles patológicos en la investigación podológica.

Este estudio, precisamente, ha pretendido servir de inspiración para la realización de otros estudios futuros similares, en los que se mejoren los tamaños muestrales y se analicen las relaciones con otros factores que den lugar a análisis más exhaustivos y hallazgos de mayor expectro en relación con la temática estudiada.

La ventaja que representan las "www" para la comunicación abierta entre científicos provocará que más y más investigación del comportarniento en relación con la podología se haga a través de Internet y que los resultados puedan influir positivamente en el aumento de las conductas preventivas para el mantenimiento de la salud del pie.

La prevención de la salud del pie y la promoción de conductas saludables relacionadas con los hábitos de conducta contribuirán a que la población se dote de los recursos necesarios para alcanzar una vida sana, autónoma y alejada de situaciones de dependencia.

\section{BIBLIOGRAFÍA}

1. Ramos, J. Detección Precoz y confirmación diagnóstica de alteraciones podológicas en la población escolar. (Tesis doctoral), 2007; Universidad de Sevilla, Sevilla.

2. Jeffcoate, W.J., \& Harding, K.G. Diabetic foot ulcers. Lancet, 2003; 362 (9398): 1858.

3. O'Donnel, T. Salud y promoción de la salud. En W. A. Turner, M.A Merriman. (Eds). Habilidades Clínicas para el tratamiento del pie. Madrid: Elsevier. 2007.

4. García-Mira R, Goluboff M, García-Fontán C, Romay J.. La experiencia perceptiva de la cuidad: diferencias en la estructuración cognitiva de peatones y pasajeros. Encuentros en Psicología Social 2004; 2 (1): 256-260.

5. Garcia-Mira R, Goluboff M. The perception of urban space from two different viewpoints: pedestrians and automobile passengers. In: Garcia-Mira, R., Uzzell, D.L., Real, J.E., \& Romay, J. (eds.). Housing, space and quality of life, Ashgate; 2005: 7-16.

6. Waxman, R., Woodburn, H., Powell, M., Woodburn, J., Blackburn, S., \& Helliwell, P. FOOTSTEP: a randomized controlled trial investigating the clinical and cost effectiveness of a patient self-management program for basic foot care in the elderly. Journal Of Clinical Epidemiology, 2003; 56 (11): 1092-1099.

7. Barr, E., Browning, C., Lord, S., Menz, H., \& Kendig, H. Foot and leg problems are important determinants of functional status in community dwelling older people. Disability And Rehabilitation, 2005; 27(16): 917-923.

8. Reiber, G.E. Epidemiology of foot ulcers and amputations in the diabetic foot. In J.H. Bowker., M.A. Pfeiffer., (Eds). The Diabetic Foot. St Louis: Mosby, 2001.

9. Corbett, C. A randomized pilot study of improving foot care in home health patients with diabetes. The Diabetes Educator, 2003; 29(2): 273-282.

10. López, D., García-Mira, R., Alonso, F., López L. Análisis del Perfil y Estilo de Vida de las personas con Patologías en los Pies. Revista Internacional de Ciencias Podológicas, 2010; 4 (2) : 45-70.

11. Yamakawa, M., Makimoto, K. Positive experiences of type 2 diabetes in Japanese patients: an exploratory qualitative study. Int J Nurs Stud., 2008; 45(7): 1032-41.

12. Wikblad, K.F. Patient perspectives of diabetes care and education, Journal of Advanced Nursing, 1991; 16 (7): 837-844.

13. Adolfssonab, E.A., Starrinc, B., Smidea, B., Wikblada, K. Type 2 diabetic patients' experiences of two different educational approaches-A qualitative study, 2008; 45, (7): 986-994. 
14. Campbell, R., Pound, P., Pope, C., Britten, N., Pill, R., Morgan, M., \& Donovan, J. Evaluating metaethnography: a synthesis of qualitative research on lay experiences of diabetes and diabetes care. Soc Sci Med, 2003; 6(4): 671-84.

15. Morris, C., Liabo, K., Wright, P., \& Fitzpatrick, R. Development of the Oxford ankle foot questionnaire: finding out how children are affected by foot and ankle problems. Child: Care, Health And Development, 2007; 33(5): 559-568.

16. Palisano, R. J., Tieman, B. L., Walter, S. D., Bartlett, D. J., Rosenbaum, P. L., Russell, D. \& Hanna, S. E. Effect of environmental setting on mobility methods of children with cerebral palsy. Developmental Medicine and Child Neurology, 2003; 45, 113-120.

17. Blain H, Jeandel C. Prevención y Envejecimiento. En: Herbaux, I., Blain, H., \& Jeandel, C. (Eds), Podología Geriátrica. Badalona : Paidotribo, 2007 p. 14-40.

18. Risso A. Experimentos Psicológicos a traves de Internet. Anuario de Psicología 32(2), 2001; $109-116$.

19. Risso A. Metodología de Investigación de las WWW. Metodología de las Ciencias del comportamiento, 2002; 487-491.

20. Estudio general de medios (EGM) de la Asociación para la investigación de los medios de comunicación. (2009). Disponible en: http://download.aimc.es/aimc/02egm/resumegm309.pdf

21. Chesterton, L., Barlas, P., Foster, N., Baxter, G., Wright, C. Gender differences in pressure pain threshold in healthy humans. Pain, 2003; 101:259-266.

22. Wise, E., Price, D., Myers, C., Heft, M., Robinson, M. Gender role expectations of pain: relationship to experimental pain perception. Pain, 2002; 96:335-342.

23. Akhtar, S., Choudry, Q., Kumar, R. Incorrectly fitting footwear and associated foot problems. Journal of Bone and Joint Surgery, 2006; 90 (3): 495-496.

24. Álvarez-Calderón, O., Alonso, F., López, D., Gómez, B., Sánchez, R. Análisis del calzado en una población mayor de 60 años. Revista Internacional de Ciencias Podológicas, 2008; 2 (1), 19-26.

25. Kerrigan, D.C., Franz, J.R., Keenan, G.S., Dicharry, J., Della, U., \& Wilder, R.P.The effect of running shoes on lower extremity joint torques. Journal American Academy of Physical Medicine and Rehabilitation, 2009; 1(12), 1058-63.

26. Teixeira, E.R., \& Figueiredo, N.M.A. O desejo e a necessidade no cuidado com o corpo: uma perspectiva estética na prática de enfermagem. Rio de Janeiro: UFF, 2001.

27. Mokdad, A.H., Marks, J.S., Stroup, D.N., Gerberding, J.L. Actual Causes of Death in the United States, 2000. JAMA. 2004; 291(10):1238-1245.

28. Estudio general de medios (EGM) de la Asociación para la investigación de los medios de comunicación. (2010). Disponible en: http://download.aimc.es/aimc/03internet/macro2009.pdf

29. Ruiz, J. La Condición Física como Determinante de Salud en Personas Jóvenes. (Tesis Doctoral). Universidad de Granada, Granada. 2007. 